\title{
Immunological biomarkers of allergic bronchopulmonary aspergillosis in patients with asthma
}

\author{
${ }^{1}$ Kozlova Y, ${ }^{2}$ Frolova E, ${ }^{2}$ Uchevatkina A, ${ }^{2}$ Filippova L, ${ }^{1}$ Sobolev A, ${ }^{1}$ Nikitin $0,{ }^{1}$ Klimko N. \\ ${ }^{1}$ North Western State Medical University, Saint-Petersburg, Russia \\ ${ }^{2}$ Kashkin Research Institute of Medical Mycology, North Western State Medical University, Saint-Petersburg, Russia
}

\section{Objectives}

To study the immunological biomarkers of allergic bronchopulmonary aspergillosis (ABPA) in patients with bronchial asthma.

\section{Methods}

In prospective study were included 44 adult patients ( $\mathrm{Me}$ 43 years) with severe asthma. The control group consisted of 12 healthy people, matched by age and sex, without a history of allergic diseases (Me - 33 years). The severity of asthma and level of symptoms control were determined with GINA 2016 criteria. The pulmonary function was assessed with FEV1 and FVC. An Asthma Control Test (AST) questionnaire was used. Allergy examination included skin testing with $A$. fumigatus allergen («Allergopharma», Germany), determination of the serum total lgE level («Polygnost», Russia) and specific $\lg E$ (slgE) for fungal allergens («AlkorBio», Russia). Determination of the serum concentration of TARC ( «R\&D Systems», USA), TSLP («R\&D Systems», USA), IL-8 («VectorBest», Russia) was performed with ELISA.

The obtained data were processed using the software system STATISTICA 10 and presented as the median (Me) and upper and lower quartiles $(\mathrm{Lq}-\mathrm{Hq})$. Severe asthma with fungal sensitization (SAFS) and ABPA were diagnosed with $R$. Agarwal et al. 2013 criteria.

\section{Results}

SAFS (positive skin test results and fungal allergens slgE levels $(0.35$ $\mathrm{IU} / \mathrm{ml}$ ) was diagnosed in $14(32 \%)$ patients, ABPA - 13 (30\%). AST score and FVC, FEV1 were significantly lower in ABPA patients. The TSLP serum levels did not differ between ABPA patients - 12.0 $(8.8 \div 24.7), \quad$ SAFS $\quad-\quad 16.8$ $(9.7 \div 27.7), \quad$ asthma -22.8 $(14.6 \div 31.8)$ and the control group - 13,2 $(9,1 \div 22,1) \mathrm{pg} / \mathrm{ml}$. The serum TARC concentration was significantly higher in ABPA patients

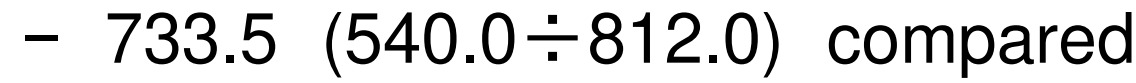
with the asthma group - 429.1 $(218.0 \div 571.3, \quad \mathrm{p}=0.001)$ and control group $202.5(195.9 \div 256.0$, $\mathrm{p}=0.001) \mathrm{pg} / \mathrm{mg}$ (Fig.1).

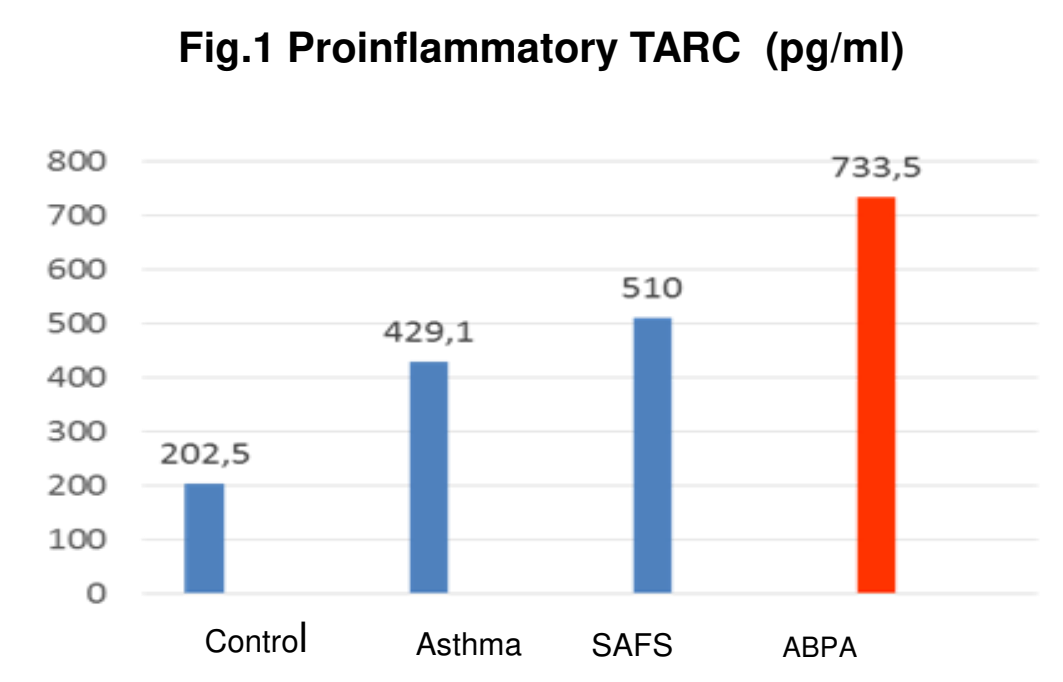

In ABPA patients serum IL-8 levels - 39,8 $(28,4 \div 54,0)$ were significantly higher than in other

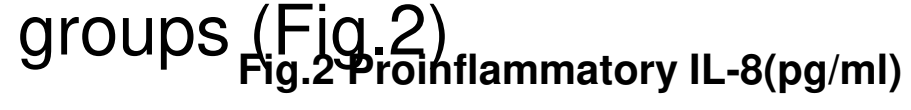

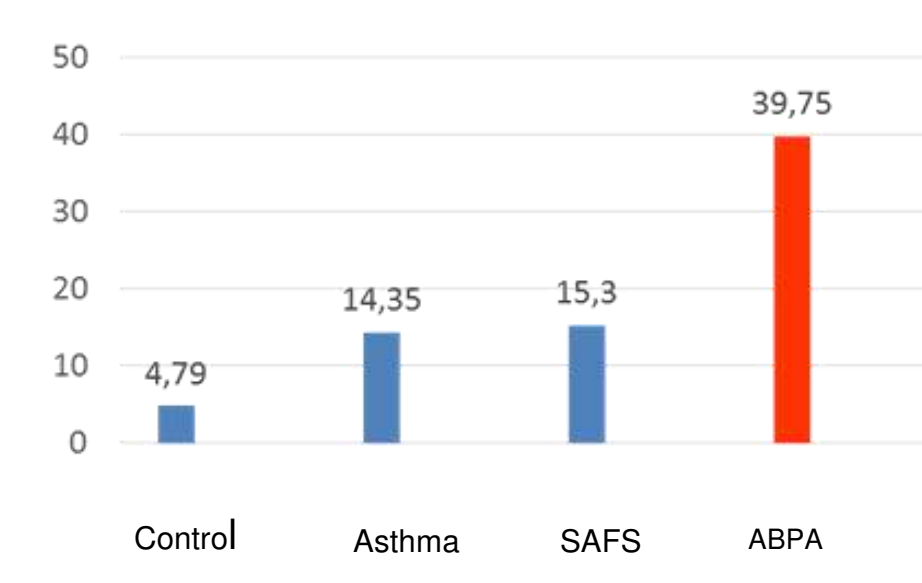

A negative correlation between TARC levels and FVC $(r=-0.47$, $p<0.05)$ and FEV1 $(r=-0.41$, $p<0.05)$ was established.

\section{Results}

The serum TARC concentration did not differ between patients with SAFS and asthma, but was significantly higher in relation to the control group $(p=0.001 ; p=$ 0.02).

Positive correlation of slgE level to $A$. fumigatus with eosinophils absolute number $(r=0.45, p$ $<0.05)$, total IgE level $(r=0.38, p$ $<0.05)$, TARC concentration $(r=$ $0,48, p<0,05)$, and IL-8 $(r=$ $0,55, \quad p<0,05)$ confirm the importance of pro-inflammatory chemokines in the development of allergic inflammation in patients with fungal sensitization (Fig.3).

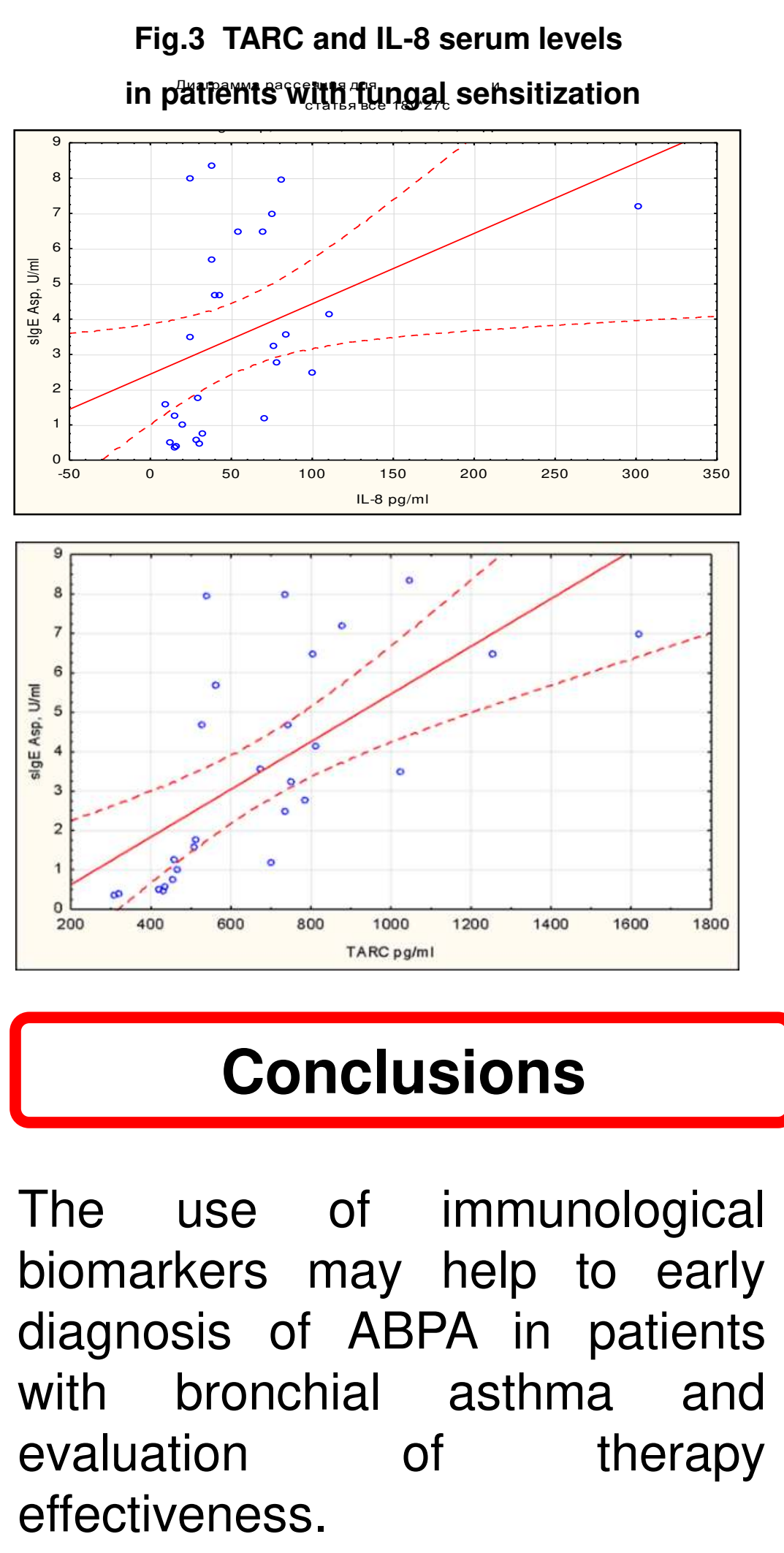

\section{P 015}

